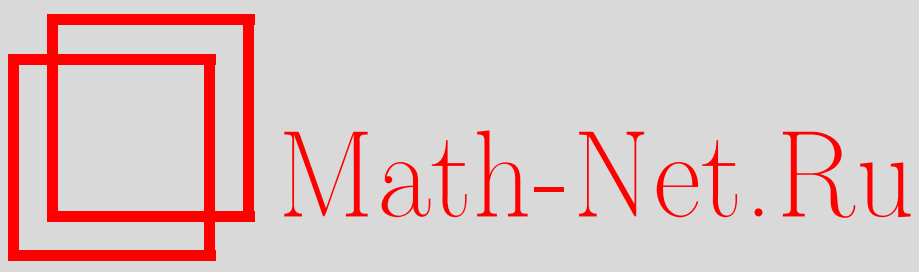

Н. Н. Холщевникова, Объединение множеств единственности кратных рядов - Уолша и тригонометрических, Матем. сб., 2002, том 193, номер 4, 135-160

DOI: https://doi.org/10.4213/sm647

Использование Общероссийского математического портала Math-Net.Ru подразумевает, что вы прочитали и согласны с пользовательским соглашением http://www . mathnet.ru/rus/agreement

Параметры загрузки:

IP: 54.209 .52 .79

26 апреля 2023 г., $15: 53: 44$ 


\title{
Объединение множеств единственности кратных рядов - Уолша и тригонометрических
}

\begin{abstract}
Для случая сходимости кратных рядов Уолша и тригонометрических по прямоугольникам исследуются вопросы об объединении множеств единственности, о формальном произведении рядов, о принципе локализации для суммируемых функций и аналогах теоремы И.И. Привалова о единственности представления функции рядом.

Библиографоия: 15 названий.
\end{abstract}

Напомним результаты о единственности кратных рядов Уолша и кратных тригонометрических рядов для случая сходимости по прямоугольникам, на которые будем ссылаться в дальнейшем.

В работах В. А. Скворцова [1] и Х. О. Мовсисяна [2] доказана

Теорема А (Скворцов, Мовсисян). Пусть кратный ряд Уолша сходится по прямоугольникам всюду, кроме, быть может, счетного множества, $к$ конечной суммируемой функции $f$. Тогда этот ряд является рядом Фурье функиии $f$.

Из теоремы А следует, что счетное множество является множеством единственности кратных рядов Уолша. В 1989 г. С. Ф. Лукомский [3] доказал, что не только счетные множества будут множествами единственности. В частности, справедливы:

Teорема В (Лукомский). Пусть $E \subset[0,1)^{d-1}(d \geqslant 2)$. Множество $E \times[0,1)$ есть множество единственности для d-кратного ряда Уолша тогда и только тогда, когда Е есть множество единственности для $(d-1)$-кратного ряда Уолша.

Tеорема С (Лукомский). Пусть $E \subset[0,1)^{d}(d \geqslant 2), E_{x_{2}, \ldots, x_{d}}=\left\{x_{1}:\right.$ $\left.\left(x_{1}, x_{2}, \ldots, x_{d}\right) \in E\right\},\left(x_{2}, \ldots, x_{d}\right) \in[0,1)^{d-1}, A=\left\{\left(x_{2}, \ldots, x_{d}\right): E_{x_{2}, \ldots, x_{d}}\right.$ более чем счетно $\}$. Если $A$-множество единственности для $(d-1)$-кратного ряда Уолиа, то Е-множество единственности для d-кратного ряда Уолwa.

Отсюда, в частности, следует, что множество $E \subset[0,1)^{d}$, состоящее из конечного или счетного числа частей гиперплоскостей, является множеством единственности.

Работа вьполнена при финансовой поддержке Российского фонда фундаментальных исследований (грант № 99-01-00355).

(C) Н.н. Холшевникова 2002 
Это редкий случай, когда результаты единственности для рядов Уолша опередили соответствующие результаты для тригонометрических рядов. До 1990 г. было неизвестно, является ли пустое множество множеством единственности для кратных тригонометрических рядов (для сходимости по прямоугольникам) в случае размерности большей двух. Для двойных тригонометрических рядов Дж.М. Эш и Г.В. Вэлланд в 1972 г. [4] доказали, что пустое множество и одноточечное множество являются множествами единственности, но будет ли также и двухточечное множество множеством единственности было неизвестно. В 1990 г. Ш.Т. Тетунашвили [5] доказал, что любое счетное множество является множеством единственности для $d$-кратного тригонометрического ряда. Ш. Т. Тетунашвили также доказаны для кратных тригонометрических рядов теоремы в духе теорем А, В и С.

Фундаментальным структурным результатом для множеств единственности тригонометрических рядов является полученная Н.К. Бари в 1923 году

ТЕОРЕМА БАРИ. Объединение счетного числа замкнутых множеств единственности является мнохсеством единственности.

Для рядов по системе Уолша в нумерации Пэли аналог теоремы Бари в случае объединения конечного числа замкнутых множеств единственности доказал А. А. Шнейдер в 1949 г., а для счетного объединения - В. Р. Вэйд в 1971 г. В доказательствах этих теорем существенно используются теоремы о формальном произведении и принцип локализации, которые в обшем не выполняются для кратных рядов. Сужая классы кратных рядов Уолша и кратных тригонометрических рядов таким образом, чтобы поведение рядов этих классов было подобно поведению одномерных рядов, мы получим с их помощью ряд результатов об объединении множеств единственности для кратных рядов. Сформулируем один из них.

Tеорема $9^{\prime}$. Пусть $E_{k} \subset(0,1)^{d} \quad(k=1,2,3, \ldots)$ - замкнутые множества единственности меры 0 для d-кратных рядов Уолиа (для сходимости по прямоугольникам). Тогда $E=\bigcup_{k=1}^{\infty} E_{k}$ тоже является множеством единственности.

Заметим, что по сравнению с одномерным случаем в этой теореме два дополнительных условия: $E_{k} \subset(0,1)^{d}$, а не $E_{k} \subset[0,1)^{d}$, и $E_{k}$ меры 0 , которые обсуждаются в дальнейшем.

\section{§1. Кратные ряды Уолша}

Приведем теперь необходимые определения и обозначения.

Обозначим через $Q_{2}$ множество двоично-рациональных чисел из отрезка $[0,1]$. Функции Уолша в нумерации Пэли определяются следуюшим образом (см., например, $[6 ;(1.2 .12)])$ :

$$
w_{n}(x)=(-1)^{\sum_{i=0}^{k} \varepsilon_{i} x_{i}}, \quad n=0,1,2, \ldots ; \quad x \in[0,1),
$$

где $\left\{x_{i}\right\}$ - последовательность знаков двоичного разложения числа $x$ (т.е. $x=$ $\sum_{i=0}^{\infty} x_{i} 2^{-i-1}$, где $x_{i}=0$ или 1$)$, причем для $x \in Q_{2}$ берется разложение с конечным числом единиц; а $\varepsilon_{i}$ определены равенством $n=\sum_{i=0}^{k} \varepsilon_{i} 2^{i}(k=k(n))$, где $\varepsilon_{i}=0$ или $1(i=0,1, \ldots, k)$. 
Если $n=\sum_{i=0}^{k} \varepsilon_{i} 2^{i}$ и $\nu=\sum_{i=0}^{k} \eta_{i} 2^{i}$, где $\varepsilon_{i}, \eta_{i}=0$ или 1 , то по определению

$$
n \dot{+} \nu=\sum_{i=0}^{k} \theta_{i} 2^{i}, \quad \text { где } \theta_{i}=\varepsilon_{i}+\eta_{i} \quad(\bmod 2)(i=0,1, \ldots, k) .
$$

Равенство (см. [6; (1.2.17)])

$$
w_{n}(x) \cdot w_{\nu}(x)=w_{n \dot{+} \nu}(x),
$$

вытекающее из (1) и (2), позволяет, в частности, определить формальное произведение ряда по системе Уолша на полином.

Рассмотрим кратные ряды Уолша и сходимость по прямоугольникам: $d$-кратный ряд Уолша

$$
\sum_{k_{1}=0, \ldots, k_{d}=0}^{\infty} a_{k_{1} \ldots k_{d}} w_{k_{1}}\left(x_{1}\right) \cdots w_{k_{d}}\left(x_{d}\right)
$$

сходится по прямоугольникам в точке $\left(t_{1}, \ldots, t_{d}\right)$ к числу $s$, если его частные суммы

$$
S_{N_{1} \ldots N_{d}}\left(t_{1}, \ldots, t_{d}\right)=\sum_{k_{1}=0}^{N_{1}} \ldots \sum_{k_{d}=0}^{N_{d}} a_{k_{1} \ldots k_{d}} w_{k_{1}}\left(t_{1}\right) \cdots w_{k_{d}}\left(t_{d}\right)
$$

стремятся к $s$, когда $\min \left(N_{1}, \ldots, N_{d}\right) \rightarrow \infty$.

Далее под сходимостью кратного ряда везде понимаем сходимость по прямоугольникам.

Через $\mathscr{K}_{d}$ обозначим класс $d$-кратных рядов Уолша (4) таких, что для каждого $1 \leqslant i \leqslant d$

$$
\lim _{1 \leqslant j \leqslant d} \sum_{N_{j} \rightarrow \infty}^{N_{1}} \ldots \sum_{k_{1}=0}^{N_{i-1}} \sum_{k_{i-1}=0}^{N_{i+1}} \cdots \sum_{k_{i+1}=0}^{N_{d}} a_{k_{1} \ldots k_{i-1} N_{i} k_{i+1} \ldots k_{d}} \prod_{\substack{r=1 \\ r \neq i}}^{d} w_{k_{r}}\left(x_{r}\right)=0
$$

для всех $\left(x_{1}, \ldots, x_{i-1}, x_{i+1}, \ldots, x_{d}\right) \in[0,1)^{d-1}$.

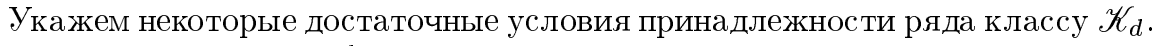

Множество $A \subset[0,1)^{d}$ назовем существенным (для $d$-кратных рядов Уолша), если на всяком координатном отрезке

$$
t_{i}=t, \quad 0 \leqslant t<1, \quad t_{j}=c_{j}(j \neq i), \text { где } c_{j} \in[0,1) \quad(i=1,2, \ldots, d),
$$

найдется точка множества $A$.

Другими словами, множество $A$ сушественное, если оно содержит $d$ поверхностей вида

$$
x_{i}=f_{i}\left(x_{1}, \ldots, x_{i-1}, x_{i+1}, \ldots, x_{d}\right) \quad(i=1,2, \ldots, d),
$$

где $f_{i}-$ произвольные функции из $[0,1)^{d-1}$ в $[0,1)$.

В частности, множество $A$, состоящее из $d$ сечений куба координатными гиперплоскостями $x_{i}=c_{i}, c_{i} \in[0,1)(i=1,2, \ldots, d)$, является сушественным.

ЛЕмма 1. Если ряд $S$ вида (4) сходится на некотором существенном множестве $A$, то $S$ - ряд класса $\mathscr{K}_{d}$. 
ДоказАтельство. Как отмечено, множество $A$ содержит $d$ поверхностей вида (6). Пусть $i, 1 \leqslant i \leqslant d$, фиксировано. Тогда ввиду сходимости $S$ на $i$-й поверхности

$$
S_{N_{1} \ldots N_{i} \ldots N_{d}}\left(x_{1}, \ldots, x_{i}, \ldots, x_{d}\right)-S_{N_{1} \ldots N_{i}-1 \ldots N_{d}}\left(x_{1}, \ldots, x_{i}, \ldots, x_{d}\right) \rightarrow 0
$$

если $\min _{i \leqslant j \leqslant d} N_{j} \rightarrow \infty$,

$$
x_{i}=f\left(x_{1}, \ldots, x_{i-1}, x_{i+1}, \ldots, x_{d}\right), \quad\left(x_{1}, \ldots, x_{i-1}, x_{i+1}, \ldots, x_{d}\right) \in[0,1)^{d-1} .
$$

Данная разность частичных сумм отличается от суммы в формуле (5) множителем $w_{i}\left(f_{i}\left(x_{1}, \ldots, x_{i-1}, x_{i+1}, \ldots, x_{d}\right)\right)$, и так как $w_{i}(t)= \pm 1$, то отсюда следует, что условие (5) выполняется. Лемма доказана.

Множество $E \subset[0,1)^{d}$ назьвается множеством единственности для $d$-кратных рядов Уолша, коротко, $U$-множеством, если из сходимости ряда вида (4) к нулю на $[0,1)^{d} \backslash E$ следует, что все коэффициенты ряда равны нулю.

Множество $E \subset[0,1)^{d}$ называется множеством единственности для класса $\mathscr{K}_{d}$, коротко, $U\left(\mathscr{K}_{d}\right)$-мно жеством, если из сходимости ряда класса $\mathscr{K}_{d}$ к нулю на $[0,1)^{d} \backslash E$ следует, что все коэффиициенты ряда равны нулю.

Множество $E \subset[0,1)^{d}$ называется $M$-множсеством $(M$-множеством для класса $\mathscr{K}_{d}$, коротко, $M\left(\mathscr{K}_{d}\right)$-мно жеством $)$, если оно не является $U$-множеством $\left(U\left(\mathscr{K}_{d}\right)\right.$-множеством $)$.

Обозначим также через $U, U\left(\mathscr{K}_{d}\right), M, M\left(\mathscr{K}_{d}\right)$ семейства соответствующих множеств.

Из определений следует, что $U \subset U\left(\mathscr{K}_{d}\right), M\left(\mathscr{K}_{d}\right) \subset M$, т.е. всякое $U$-множество является $U\left(\mathscr{K}_{d}\right)$-множеством и всякое $M\left(\mathscr{K}_{d}\right)$-множество является $M$-множеством.

Лемма 2. Пусть $E \in U\left(\mathscr{K}_{d}\right)$ и $A$ - существенное множество такое, ито $E \cap A=\varnothing$. Тогда $E \in U$.

ДокАЗАТЕЛЬСТво следует непосредственно из определений класса $\mathscr{K}_{d}, U$ и $U\left(\mathscr{K}_{d}\right)$-множеств и леммы 1.

Ряды $S_{1}$ и $S_{2}$ называются равносходящимися на множсестве $E$, если их разность $S_{1}-S_{2}$ сходится к нулю на $E$.

Если $E \subset[0,1)^{d}$, то через $\chi_{E}\left(x_{1}, \ldots, x_{d}\right)$ обозначается характеристическая функция множества $E$, т.е. функция, равная 1 на $E$ и 0 вне $E$.

Для простоты будем дальше рассматривать двойные ряды Уолша, поясняя при необходимости результаты для $d$-мерного случая.

Пусть $p(x, y)$ - полином по системе Уолша

$$
p(x, y)=\sum_{l=0}^{r} \sum_{s=0}^{p} c_{l s} w_{l}(x) w_{s}(y) .
$$

Равенство (3) позволяет определить формальное произведение двойного ряда $S$ по системе Уолша

$$
S=\sum_{k, m=0}^{\infty} a_{k m} w_{k}(x) w_{m}(y)
$$


на полином $p(x, y)$ как ряд

$$
\widetilde{S}=\sum_{k, m=0}^{\infty} \widetilde{a}_{k m} w_{k}(x) w_{m}(y), \quad \text { где } \widetilde{a}_{k m}=\sum_{l=0}^{r} \sum_{s=0}^{p} c_{l s} a_{k \dot{+} l, m \dot{+} s} .
$$

Нетрудно видеть, что если $f(x, y)$ - суммируемая функция и $S(f)$ - ее ряд Фурье-Уолша, то формальное произведение $S(f)$ на $p(x, y)$ есть ряд Фурье-Уолша функции $p(x, y) \cdot f(x, y)$ :

$$
\widetilde{S}(f)=S(p \cdot f)
$$

Условия (5) принадлежности классу $\mathscr{K}_{d}$ для двойного ряда (7) выглядят следующим образом

$$
\lim _{\min (N, M) \rightarrow \infty} \sum_{k=0}^{N} a_{k M} w_{k}(x)=0, \quad x \in[0,1),
$$

$$
\lim _{\min (N, M) \rightarrow \infty} \sum_{m=0}^{M} a_{N m} w_{m}(y)=0, \quad y \in[0,1) .
$$

Обозначим через $\mathscr{P}$ множество прямоугольников вида $\Pi=[\alpha, \beta) \times[\gamma, \delta)$, где $\alpha, \beta, \gamma, \delta \in Q_{2}$.

Как известно (см., например, [6; п. 1.3.2]), если $\alpha, \beta, \gamma, \delta \in Q_{2}$, то характеристические функции $\chi_{[\alpha, \beta)}(x)$ и $\chi_{[\gamma, \delta)}(y)$ являются полиномами по системе Уолша:

$$
\chi_{[\alpha, \beta)}(x)=\sum_{l=0}^{r} c_{l} w_{l}(x), \quad \chi_{[\gamma, \delta)}(y)=\sum_{s=0}^{p} b_{s} w_{s}(y) .
$$

Так как $\chi_{\Pi}(x, y)=\chi_{[\alpha, \beta)}(x) \cdot \chi_{[\gamma, \delta)}(y)$, то в случае, когда $\Pi \in \mathscr{P}$,

$$
\chi_{\Pi}(x, y)=\sum_{l=0}^{r} \sum_{s=0}^{p} c_{l} b_{s} w_{l}(x) w_{s}(y)
$$

Теорема 1. Пусть $S$ - двойной ряд по системе Уолша класса $\mathscr{K}_{2}$. Для любого прямоугольника $\Pi \in \mathscr{P}$ существует ряд $\widetilde{S}$ класса $\mathscr{K}_{2}$, равносходящийся с $S$ на П и сходящийся $\kappa$ нулю вне П ( $\widetilde{S}$ есть формальное произведение $S$ на полином $\left.\chi_{\Pi}(x, y)\right)$.

ДоказАтЕЛьство. Пусть $\widetilde{S}$ - формальное произведение $S$ на полином $\chi_{\text {П }}(x, y)$ (см. (12)) и $q$ таково, что $p<2^{q}, r<2^{q}$. Рассмотрим разность частичных сумм рядов $S$ и $\widetilde{S}$ порядка $N, M$ :

$$
\begin{aligned}
R_{N M}(x, y) & =S_{N M}(x, y)-\widetilde{S}_{N M}(x, y) \\
& =\sum_{k=0}^{N} \sum_{m=0}^{M} a_{k m} w_{k}(x) w_{m}(y)-\sum_{k=0}^{N} \sum_{m=0}^{M} \widetilde{a}_{k m} w_{k}(x) w_{m}(y)
\end{aligned}
$$

при $\min (N, M) \geqslant 2^{q}-1$. 
Заметим, что

$$
\nu \cdot 2^{q}-1 \leqslant N<(\nu+1) 2^{q}-1, \quad \mu \cdot 2^{q}-1 \leqslant M<(\mu+1) 2^{q}-1
$$

для некоторых натуральных $\nu=\nu(N), \mu=\mu(M)$.

Из формулы сложения (2) вытекает, что если $l<2^{q}$, то $l+k$ пробегает все значения $0,1, \ldots, \nu \cdot 2^{q}-1$, когда $k$ пробегает значения от 0 до $\nu \cdot 2^{q}-1$. Пользуясь этим результатом и формулой $(3)$, получим

$$
\begin{aligned}
\widetilde{S}_{\nu \cdot 2^{q}}-1, \mu \cdot 2^{q}-1 & (x, y)=\sum_{k=0}^{\nu \cdot 2^{q}-1} \sum_{m=0}^{\mu \cdot 2^{q}-1} \widetilde{a}_{k m} w_{k}(x) w_{m}(y) \\
& =\sum_{k=0}^{\nu \cdot 2^{q}-1} \sum_{m=0}^{\mu \cdot 2^{q}-1} \sum_{l=0}^{r} \sum_{s=0}^{p} c_{l} b_{s} a_{k \dot{+} l, m \dot{+} s} w_{k}(x) w_{m}(y) w_{l}^{2}(x) w_{s}^{2}(y) \\
& =\sum_{l=0}^{r} c_{l} w_{l}(x) \sum_{s=0}^{p} b_{s} w_{s}(y) \sum_{k=0}^{\nu \cdot 2^{q}-1} \sum_{m=0}^{\mu \cdot 2^{q}-1} a_{k \dot{+} l, m \dot{+} s} w_{k \dot{+} l}(x) w_{m+s}(y) \\
& =\chi_{\Pi}(x, y) \cdot S_{\nu \cdot 2^{q}-1, \mu \cdot 2^{q}-1}(x, y) .
\end{aligned}
$$

Таким образом,

$$
\widetilde{S}_{\nu \cdot 2^{q}-1, \mu \cdot 2^{q}-1}(x, y)= \begin{cases}S_{\nu \cdot 2^{q}-1, \mu \cdot 2^{q}-1}(x, y), & (x, y) \in \Pi, \\ 0, & (x, y) \in[0,1)^{2} \backslash \Pi .\end{cases}
$$

Тогда для $(x, y) \in \Pi$

$$
\begin{aligned}
R_{N M}(x, y)= & \sum_{k=0}^{N} \sum_{m=\mu \cdot 2^{q}}^{M}\left(a_{k m}-\widetilde{a}_{k m}\right) w_{k}(x) w_{m}(y) \\
& +\sum_{k=\nu \cdot 2^{q}}^{N} \sum_{m=0}^{\mu \cdot 2^{q}}\left(a_{k m}-\widetilde{a}_{k m}\right) w_{k}(x) w_{m}(y) .
\end{aligned}
$$

Из (10), (11) и (13) следует, что

$$
\lim _{\min (N, M) \rightarrow \infty}\left(S_{N M}(x, y)-S_{\nu \cdot 2^{q}-1, \mu \cdot 2^{q}-1}(x, y)\right)=0, \quad(x, y) \in[0,1)^{2} .
$$

Покажем, что для ряда $\widetilde{S}$ тоже выполняется условие (10). Действительно,

$$
\begin{aligned}
\sum_{k=0}^{N} \widetilde{a}_{k M} w_{k}(x)= & \sum_{k=0}^{N} \sum_{l=0}^{r} \sum_{s=0}^{p} c_{l s} a_{k \dot{+} l, m \dot{+} s} w_{k}(x) w_{l}^{2}(x) \\
= & \sum_{l=0}^{r} \sum_{s=0}^{p} c_{l s} w_{l}(x) \sum_{k=0}^{N} a_{k \dot{+} l, m \dot{+} s} w_{k \dot{+} l}(x) \\
= & \sum_{l=0}^{r} \sum_{s=0}^{p} c_{l s} w_{l}(x) \sum_{k=0}^{\nu \cdot 2^{q}-1} a_{k, m \dot{+} s} w_{k}(x) \\
& +\sum_{l=0}^{r} \sum_{s=0}^{p} c_{l s} w_{l}(x) \sum_{k=\nu \cdot 2^{q}}^{N} a_{k \dot{+} l, m \dot{+} s} w_{k \dot{+} l}(x) .
\end{aligned}
$$


Так как $s \leqslant p, l \leqslant r$, то $m \dot{+} s \rightarrow \infty$, если $m \rightarrow \infty$, и $k \dot{+} l \rightarrow \infty$, если $k \rightarrow \infty$. Тогда из последних формул и условия (10) для ряда $S$ вытекает, что для ряда $\widetilde{S}$ тоже выполняется условие (10). Аналогично можно показать, что для ряда $\widetilde{S}$ выполняется условие (11). Отсюда следует, что ряд $\widetilde{S}$ является рядом класса $\mathscr{K}_{2}$. Тогда, аналогично формуле (16) для ряда $S$, для ряда $\widetilde{S}$ имеем

$$
\lim _{\min (N, M) \rightarrow \infty}\left(\widetilde{S}_{N M}(x, y)-\widetilde{S}_{\nu \cdot 2^{q}-1, \mu \cdot 2^{q}-1}(x, y)\right)=0, \quad(x, y) \in[0,1)^{2} .
$$

Из (15), (16) и (17) следует, что

$$
\lim _{\min (N, M) \rightarrow \infty} R_{N M}(x, y)=0, \quad(x, y) \in \Pi,
$$

т.е. ряды $S$ и $\widetilde{S}$ равносходятся на П, а из (14) и (17) следует, что

$$
\lim _{\min (N, M) \rightarrow \infty} \widetilde{S}_{N M}(x, y)=0, \quad(x, y) \in[0,1)^{2} \backslash \Pi .
$$

Теорема доказана.

Доказанная теорема позволяет получить принцип локализации для рядов $Ф у-$ рье-Уолша класса $\mathscr{K}_{2}$, а именно справедлива

Tеорема 2. Пусть $f(x, y)$ и $g(x, y)$ - суммируемъе функции, ряды $Ф у$ рве-Уолиа которых $S(f)$ и $S(g)$ принадлежат классу $\mathscr{K}_{2}$. Если $f$ и $g$ совпадают на некотором открытом множестве $G$, то $S(f)$ и $S(g)$ равносходятся на $G$.

ДокАЗАтЕльСтво. Пусть $\left(x_{0}, y_{0}\right) \in G$, и $\Pi \in \mathscr{P}$ - такой прямоугольник, что $\left(x_{0}, y_{0}\right) \in \Pi \subset G$. В силу теоремы 1 формальное произведение ряда $S(f)$ на полином $\chi_{\Pi}(x, y)$ - ряд $\widetilde{S}_{1}$ - равносходится с $S(f)$ на П, при этом ввиду $(9) \widetilde{S}_{1}$ есть ряд Фурье функции $\chi_{\Pi}(x, y) \cdot f(x, y)$. Аналогично, формальное произведение ряда $S(g)$ на полином $\chi_{\Pi}(x, y)$ - ряд $\widetilde{S}_{2}$ - равносходится с $S(g)$ на П, причем $\widetilde{S}_{2}$ есть ряд Фурье функции $\chi_{\Pi}(x, y) \cdot g(x, y)$. Но $f$ и $g$ совпадают на П, поэтому $\widetilde{S}_{1}=\widetilde{S}_{2}$. Отсюда вытекает, что $S(f)$ и $S(g)$ равносходятся на П. Следовательно, $S(f)$ и $S(g)$ равносходятся и на $G$. Теорема доказана.

В 1923 г. И.И. Привалов [7] доказал следуюшую теорему:

если тригонометрический ряд сходится $к$ конечной суммируемой функиии $f$ всюду, кроме, быть может, некоторого замкнутого $U$-множества, то этот ряд есть ряд Фурье функиии $f$.

Аналог теоремы Привалова для системы Уолша получен В. А. Скворцовым [8] в 1973 г. Докажем некоторый аналог теоремы Привалова для кратных рядов Уолша класса $\mathscr{K}_{2}$.

ТЕОРема 3. Пусть ряд $S$ класса $\mathscr{K}_{2}$ сходится по прямоугольникам всюду, за исключением, быть может, замкнутого $U\left(\mathscr{K}_{2}\right)$-множсества $E$, к конечной суммируемой функции $f$, причем ряд Фурье $S(f)$ функции $f$ тоже класса $\mathscr{K}_{2}$. Тогда $S=S(f)$. 
ДокаЗАТЕльство. Пусть $\left(x_{0}, y_{0}\right) \notin E$. Тогда найдется прямоугольник П $\in \mathscr{P}$ такой, что $\left(x_{0}, y_{0}\right) \in \Pi, \Pi \cap E=\varnothing$. Ряд $\widetilde{S}$, являющийся формальным произведением ряда $S$ на полином $\chi_{\Pi}(x, y)$, равносходится с $\chi_{\Pi} \cdot S$ в силу теоремы 1. Следовательно, $\widetilde{S}$ всюду сходится к суммируемой функции $\chi_{\Pi} \cdot f$. По теореме А Скворцова-Мовсисяна ряд $\widetilde{S}$ является рядом Фурье-Уолша функции $\chi_{\Pi} \cdot f: \widetilde{S}=S\left(\chi_{\Pi} \cdot f\right)$. Но в силу (9) ряд $S\left(\chi_{\Pi} \cdot f\right)$ совпадает с формальным произведением $S(f)$ на полином $\chi_{\Pi}$, т.е. рядом $\widetilde{S}(f)$ и, значит, $\widetilde{S}=\widetilde{S}(f)$. Так как $S(f)$ - ряд класса $\mathscr{K}_{2}$, то $\widetilde{S}(f)$ равносходится с $\chi_{\Pi} \cdot S(f)$ и, следовательно, $S(f)$ сходится к $f$ на П. Отсюда вытекает, что $S(f)$ сходится к $f$ всюду вне $E$. Таким образом, $S-S(f)$ сходится к нулю всюду вне $E$, и так как $E$ является $U\left(\mathscr{K}_{2}\right)$-множеством, то $S=S(f)$. Теорема доказана.

Ядром ряда $S$ называется множество всех тех точек, в которых $S$ не сходится к нулю.

ЛЕмма 3. Если $\mathscr{E}-$ ядро ряда $S$ класса $\mathscr{K}_{2}$ и $\mathscr{E} \cap G \neq \varnothing$ для некоторого открытого множсества $G$, то найдется ряд $\widetilde{S} к л а с с а ~ \mathscr{K}_{2}$ с ядром $\mathscr{E}_{1}, \varnothing \neq$ $\mathscr{E}_{1} \subset \mathscr{E} \cap G ;$ в частности, множество $\mathscr{E} \cap G$ является $M\left(\mathscr{K}_{2}\right)$-множеством.

ДокАЗАтЕльство. Выберем $\left(x_{0}, y_{0}\right) \in \mathscr{E} \cap G$ и прямоугольник $\Pi \in \mathscr{P}$ так, что $\left(x_{0}, y_{0}\right) \in \Pi \subset G$. Ряд $\widetilde{S}$, являющийся формальным произведением $S$ на полином $\chi_{\Pi}(x, y)$, в силу теоремы 1 будет искомым. Лемма доказана.

Приведем теперь некоторые результаты из [9], с помощью которых получим теоремы об объединении $U$-множеств.

Пусть $X$ - топологическое пространство, $\mathscr{U}$ - семейство подмножеств $X$, обладающее тем свойством, что

$$
\text { если } A \in \mathscr{U} \text { и } A_{1} \subset A \text {, то } A_{1} \in \mathscr{U} \text {. }
$$

Такое семейство $\mathscr{U}$ называется наследственным.

Пусть $\mathscr{M}$ - семейство, дополнительное к $\mathscr{U}$, т.е. семейство множеств, не принадлежащих $\mathscr{U}$ :

$$
\mathscr{M}=\{A \subset X: A \notin \mathscr{U}\} .
$$

Назовем множество $A \in \mathscr{M}$ ядром, если для всякого открытого множества $G$ такого, что $A \cap G \neq \varnothing$, множество $A \cap G \in \mathscr{M}$.

Скажем, что $\mathscr{M}$ - семейство со свойством ядра, если каждое множество $E \in$ $\mathscr{M}$ содержит подмножество $A$, являюшееся ядром.

Замькание множества $E \subset X$ будем обозначать через $\bar{E}$.

Множество $N$ назовем приведенным ядром ядра $A$, если $N \subset A \subset \bar{N}$ и $N$ не является множеством первой категории на себе.

Назовем $\mathscr{M}$ семейством со свойством приведенного ядра, если всякое ядро $A \in \mathscr{M}$ содержит приведенное ядро.

ТеОРема D. Пусть $X$ - топологическое пространство, $\mathscr{U}$ - наследственное семейство подмножеств $X, \mathscr{M}$ - дополнительное $\kappa \mathscr{U}$ семейство со свойством ядра и со свойством приведенного ядра. Если $E_{n} \in \mathscr{U} u E_{n}$ замкнуты относительно своего объединения $E=\bigcup_{n=1}^{\infty} E_{n}$, то $E \in \mathscr{U}$.

Пусть $\mathscr{M}_{1}$ - подсемейство семейства $\mathscr{M}$. Скажем, что $\mathscr{M}_{1}-$ подсемейство со свойством ядра, если каждое множество $E \in \mathscr{M}_{1}$ содержит ядро, и что $\mathscr{M}_{1}-$ 
подсемейство со свойством приведенного ядра, если всякое ядро $A$ из $\mathscr{M}_{1}$ содержит приведенное ядро.

ТеОрема Е. Пусть $X$ - топологическое пространство, $\mathscr{U}$ - наследственное семейство подмнохсеств $X, \mathscr{M}$ - дополнительное $к$ U семейство, $\mathscr{M}_{1}$ - подсемейство $\mathscr{M}$ со свойством ядра и со свойством приведенного ядpа. Если $E_{n} \in \mathscr{U}, E_{n}(n \in \mathbb{N})$ замкнуты относительно своего облединения $E=\bigcup_{n=1}^{\infty} E_{n} u E \in \mathscr{U} \cup \mathscr{M}_{1}$, mo $E \in \mathscr{U}$.

Напомним, что топологическое пространство $X$ называется полным метризуемым пространством (в топологическом смысле), если на $X$ можно определить метрику, порождающую топологию пространства $X$, и относительно этой метрики $X$ - полное метрическое пространство. (Так $[0,1)^{d}$ - полное метризуемое пространство, хотя как метрическое пространство с евклидовой метрикой в $\mathbb{R}^{d}$ не является полным.)

Теорема F. Пусть $X$ - полное метризуемое пространство, $\mathscr{U}$ - наследственное семейство подмнохеств $X, \mathscr{M}$ - дополнительное $к \mathscr{U}$ семейство со свойством ядра. Если $E_{n} \in \mathscr{U}(n \in \mathbb{N}), E_{n}$ замкнуты относительно своего обгединения $E=\bigcup_{n=1}^{\infty} E_{n}$ и $E$ типа $G_{\delta}$, то $E \in \mathscr{U}$. Если, в частности, $E_{n} \in \mathscr{U}, E_{n}$ замкнуты и $E$ - замкнутое мнохсество, то $E \in \mathscr{U}$.

Теорема К. Пусть $X$ - полное метризуемое пространство, И - наследственное семейство подмножеств $X, \mathscr{M}$ - дополнительное $\kappa \mathscr{U}$ семейство со свойством ядра. Тогда если $E_{1}, E_{2} \in \mathscr{U} u$

1) $E_{1}$ и $E_{2}$ - множества типа $G_{\delta}$, a $E_{1} \cap E_{2}-$ типа $F_{\sigma}$ (в частности, nycmo)

uлu

2) $E_{1}$ - множество типа $G_{\delta}$ и $F_{\sigma}$ одновременно, mo $E_{1} \cup E_{2} \in \mathscr{U}$.

Семейство $U\left(\mathscr{K}_{2}\right)$ является наследственньм семейством, а $M\left(\mathscr{K}_{2}\right)$ - дополнительное к $U\left(\mathscr{K}_{2}\right)$ семейство - является в силу леммы 3 семейством со свойством ядра.

Отсюда и из теоремы F следует

ТЕОРЕМа 4. Пусть $E_{n}-U\left(\mathscr{K}_{2}\right)$-множества, замкнутые относительно своего обвединения $E=\bigcup_{n=1}^{\infty} E_{n}$, причем $E$ имеет тип $G_{\delta}$. Тогда $E$ тоже является $U\left(\mathscr{K}_{2}\right)$-мно жеством.

Из теоремы К и леммы 2 следует

ТЕОРЕМА 5. Пусть $E_{1}$ и $E_{2}$ являются $U$-множествами для двойных рядов Уолша, причем множество $[0,1)^{2} \backslash\left(E_{1} \cup E_{2}\right)$ содержст какое-нибудь существенное множество. Тогда если

1) $E_{1}$ и $E_{2}$ - множества типа $G_{\delta}$, а $E_{1} \cap E_{2}-$ типа $F_{\sigma}$ (в частности, nycmo)

uлu

2) $E_{1}-$ множсество типа $G_{\delta}$ и $F_{\sigma}$ одновременно, то $E_{1} \cup E_{2}$ является $U$-мнохсеством. 
Теоремы 1-5 справедливы также для $d$-кратного случая $(d>2)$.

\section{§ 2. Результаты, связанные со свойством приведенного ядра}

В. А. Скворцовьм [1] доказана теорема о том, что из сходимости двойного ряда Уолша (7) по прямоугольникам во всех точках "креста" $\{a \times[0,1)\} \cup\{[0,1) \times b\}$, за исключением, быть может, некоторого счетного множества, следует, что $\lim _{k+m \rightarrow \infty} a_{k m}=0$.

Покажем, что для рядов класса $\mathscr{K}_{2}$ поведение коэффициентов такое же.

ТЕОРема 6. Пуст для двойного ряда Уолша (7) выполнены условия

$$
\lim _{\min (N, M) \rightarrow \infty} \sum_{k=0}^{N} a_{k M} w_{k}(x)=0, \quad x \in[0,1) \backslash A,
$$

$$
\lim _{\min (N, M) \rightarrow \infty} \sum_{m=0}^{M} a_{N m} w_{m}(y)=0, \quad y \in[0,1) \backslash B
$$

где $A$ и В имеют мощность меньше континуума. Тогда

$$
\lim _{k+m \rightarrow \infty} a_{k m}=0
$$

ДоКАЗАТЕЛЬСТво. Из выполнения равенства в формуле (18) или (19) хотя бы в одной точке следует, что

$$
\lim _{\min (k, m) \rightarrow \infty} a_{k m}=0
$$

Допустим, что (20) не выполняется. Пусть, для определенности, найдется такой номер $l$ и последовательность номеров $k_{1}<k_{2}<\cdots$, что

$$
\left|a_{k_{i} l}\right|>C>0 \quad(i=1,2,3, \ldots)
$$

для некоторой постоянной $C$.

Каждая функция $w_{n}(x)$ с номером $2^{s} \leqslant n<2^{s+1}$ постоянна на полуинтервалах $\Delta_{s+1}^{2 r}=\left[\frac{2 r}{2^{s+1}}, \frac{2 r+1}{2^{s+1}}\right)$ и $\Delta_{s+1}^{2 r+1}=\left[\frac{2 r+1}{2^{s+1}}, \frac{2 r+2}{2^{s+1}}\right)$, причем принимает на них значения разных знаков $\left(r=0,1, \ldots, 2^{s}-1\right)$.

Поэтому суммы вида $\sum_{n=2^{s}}^{2^{s+1}-1} b_{n} w_{n}(x)$ на $\Delta_{s+1}^{2 r}$ и на $\Delta_{s+1}^{2 r+1}$ тоже постоянны и принимают значения разных знаков.

Полуинтервалы вида $\left[\frac{r}{2^{s}}, \frac{r+1}{2^{s}}\right)$ назьвают интервалами ранга $s$.

Пусть $K_{j} \subset \mathbb{N}$ - бесконечное множество натуральных чисел и $\Delta_{j}$ - интервал ранга $j$. Покажем, что если

$$
\left|\sum_{m=0}^{2^{j}-1} a_{k m} w_{m}(y)\right|>C>0 \quad \text { для } k \in K_{j}, \quad y \in \Delta_{j}
$$


то найдутся бесконечное множество $K_{j+1} \subset K_{j}$ и интервал $\Delta_{j+1}$ ранга $j+1$ такие, что

$$
\left|\sum_{m=0}^{2^{j+1}-1} a_{k m} w_{m}(y)\right|>C, \quad k \in K_{j+1}, \quad y \in \Delta_{j+1} \subset \Delta_{j} .
$$

Действительно, обозначим через $\Delta(k)$ ту из половин интервала $\Delta_{j}$, на которой сумма $\sum_{m=2^{j}}^{2^{j+1}-1} a_{k m} w_{m}(y)$ имеет тот же знак, что и $\sum_{m=0}^{2^{j}-1} a_{k m} w_{m}(y)$ на $\Delta_{j}$ (если эта сумма равна нулю, то возьмем, например, левую половину интервала $\Delta_{j}$ ). Тогда для бесконечного числа $k$ из $K_{j}$ интервалы $\Delta(k)$ совпадают. Ту из половин $\Delta_{j}$, с которой совпадает бесконечное число $\Delta(k)$ (если их две, то левую половину), обозначим через $\Delta_{j+1}$. Отсюда видно, что из (23) следует $(24)$.

Пусть $s$ - наименьшее натуральное число такое, что $2^{s}-1>l$. Тогда

$$
\begin{aligned}
\left|\sum_{m=0}^{2^{s}-1} a_{k m} w_{m}(y)\right| & =\left|\sum_{m=0}^{2^{s}-1} a_{k m} w_{m}(y) w_{l}(y)\right|=\left|\sum_{m=0}^{2^{s}-1} a_{k m} w_{m \dot{+} l}(y)\right| \\
& =\left|a_{k l}+\sum_{\nu=1}^{2^{s}-1} a_{k, \nu \dot{+} l} w_{\nu}(y)\right|=\left|a_{k l}+\sum_{r=1}^{s} \sum_{\nu=2^{r-1}}^{2^{r}-1} a_{k, \nu \dot{+},} w_{\nu}(y)\right| .
\end{aligned}
$$

Из (22)-(24) следует, что найдется интервал $\Delta_{s}$ ранга $s$ и бесконечное множество $K_{s} \subset\left\{k_{i}\right\}_{i=1}^{\infty}$ такие, что

$$
\left|\sum_{m=0}^{2^{s}-1} a_{k m} w_{m}(y)\right|>C, \quad k \in K_{s}, \quad y \in \Delta_{s} .
$$

Отсюда ввиду (23), (24) следует, что для $j \geqslant s$ выполняется $(24)$, причем $K_{j} \subset$ $\left\{k_{i}\right\}_{i=1}^{\infty}$. Выберем $\nu_{j} \in K_{j}$ так, чтобы $\nu_{j}>\nu_{j-1}$. Тогда имеем

$$
\left|\sum_{m=0}^{2^{j}-1} a_{\nu_{i} m} w_{m}(y)\right|>C, \quad i \geqslant j, \quad y \in \Delta_{j} \quad(j \geqslant s) .
$$

Пусть $0<C^{\prime}<C$. Покажем, что для всякого $j_{0}>s$ найдется номер $j_{1}>j_{0}$ такой, что для бесконечного числа номеров $i$

$$
\left|\sum_{m=0}^{2^{j_{1}}-1} a_{\nu_{i} m} w_{m}(y)\right|>C^{\prime}, \quad y \in \Delta_{j_{1}}^{\prime}=\Delta_{j_{1}-1} \backslash \Delta_{j_{1}} .
$$

Положим

$$
\delta_{i j}=\left|\sum_{m=2^{j}-1}^{2^{j}-1} a_{\nu_{i} m} w_{m}(y)\right|, \quad y \in \Delta_{j}
$$

Если для некоторого номера $j_{1}>j_{0}$ для бесконечного числа номеров $i$ выполняется неравенство $\delta_{i j_{1}} \geqslant \delta_{i, j_{1}+1}$, то на интервале $\Delta_{j_{1}+1}^{\prime}$ имеем

$$
\left|\sum_{m=0}^{2^{j_{1}+1}-1} a_{\nu_{i} m} w_{m}(y)\right|=\left|\sum_{m=0}^{2^{j_{1}-1}-1} a_{\nu_{i} m} w_{m}(y)\right|+\delta_{i j_{1}}-\delta_{i, j_{1}+1}>C,
$$

и для таких номеров $j_{0}$ утверждение (26) доказано. 
Пусть теперь номер $j_{0}$ таков, что для любого $j>j_{0}$

$$
\delta_{i j}<\delta_{i, j+1} \text { для всех } i, \text { начиная с некоторого } i(j) .
$$

Из $(21),(27)$ и (28) следует, что последовательности $\left\{\delta_{i j}\right\}\left(j>j_{0}\right)$ ограничены по $i$ :

$$
0 \leqslant \delta_{i j}<k(j) \quad(i \in \mathbb{N}) .
$$

Тогда можно выбрать такую последовательность $\left\{i_{r}\right\}$, что существуют конечные пределы

$$
\lim _{r \rightarrow \infty} \delta_{i_{r} j}=\delta_{j} \quad\left(j>j_{0}\right) .
$$

При этом, как следует из $(28), \delta_{j} \leqslant \delta_{j+1}\left(j>j_{0}\right)$.

Пусть $\delta=\lim _{j \rightarrow \infty} \delta_{j}$.

Рассмотрим три возможные случая: а) $\delta=0$, б) $0<\delta<\infty$, в) $\delta=\infty$.

В случае а) $\delta_{j}=0$ для $j>j_{0}$. Положим $j_{1}=j_{0}+1$. В силу (29) найдется такой номер $N$, что $\left|\delta_{i_{r} j_{1}}\right|<C-C^{\prime}$ для $r>N$. Тогда на интервале $\Delta_{j_{1}}^{\prime}$ имеем

$$
\left|\sum_{m=0}^{2^{j} 1-1} a_{k m} w_{m}(y)\right|>\left|\sum_{m=0}^{2^{j} 0-1} a_{k m} w_{m}(y)\right|-\delta_{k j_{1}}>C^{\prime}
$$

для $k \in\left\{\nu_{i_{r}}\right\}_{r}>N$.

В случае б) выберем такое $q$, что

$$
\frac{2}{3} \delta<\delta_{q} \leqslant \delta_{q+1} \leqslant \delta_{q+2} \leqslant \delta, \quad q>j_{0}
$$

Пусть $N$ таково, что при $r \geqslant N$

$$
\frac{2}{3} \delta<\delta_{i_{r} p}<\frac{4}{3} \delta \quad(p=q, q+1, q+2) .
$$

Тогда

$$
\begin{aligned}
\left|\sum_{m=0}^{2^{q+2}-1} a_{k m} w_{m}(y)\right| & =\left|\sum_{m=0}^{2^{q-1}-1} a_{k m} w_{m}(y)\right|+\delta_{k q}+\delta_{k, q+1}-\delta_{k, q+2} \\
& >C+\frac{2}{3} \delta+\frac{2}{3} \delta-\frac{4}{3} \delta=C
\end{aligned}
$$

при $y \in \Delta_{q+2}^{\prime}$ и $k \in\left\{\nu_{i_{r}}\right\}_{r} \geqslant N$, т.е. выполняется (26) для $j_{1}=q+2$.

В случае в) покажем, что найдется номер $n$ такой, что

$$
\delta_{1}+\delta_{2}+\cdots+\delta_{n}>\delta_{n+1}-\left(C-C^{\prime}\right)
$$

Из $(21),(27)$ и $(29)$ следует, что $\delta_{j}=o\left(2^{j}\right)$. Положим $\varepsilon_{j}=\frac{\delta_{j}}{2^{j}}$. Пусть $p>j_{0}$ таково, что $\varepsilon_{j}>0$, начиная с номера $p ; \lim _{j \rightarrow \infty} \varepsilon_{j}=0$. Имеем

$$
\begin{aligned}
& \delta_{p}+\delta_{p+1}+\cdots+\delta_{n}=\varepsilon_{p} \cdot 2^{p}+\varepsilon_{p+1} \cdot 2^{p+1}+\cdots+\varepsilon_{n} \cdot 2^{n} \\
& \quad \geqslant \min _{p \leqslant r \leqslant n}\left\{\varepsilon_{r}\right\} \cdot\left(2^{p}+2^{p+1}+\cdots+2^{n}\right)=\min _{p \leqslant r \leqslant n}\left\{\varepsilon_{r}\right\} \cdot\left(2^{n+1}-2^{p}\right) .
\end{aligned}
$$


Выберем $n>j_{0}$ таким, чтобы $\min _{p \leqslant r \leqslant n}\left\{\varepsilon_{r}\right\} \cdot 2^{p}<C-C^{\prime}$ и $\varepsilon_{n+1}<\min _{p \leqslant r \leqslant n}\left\{\varepsilon_{r}\right\}$. Тогда выполняется (30).

Ввиду (29) найдется номер $N$ такой, что

$$
\delta_{i_{r} p}+\cdots+\delta_{i_{r} n}>\delta_{i_{r}, n+1}-\left(C-C^{\prime}\right) \text { при } r \geqslant N
$$

Отсюда и из (25) следует, что

$$
\begin{aligned}
\left|\sum_{m=0}^{2^{n+1}-1} a_{k m} w_{m}(y)\right| & =\left|\sum_{m=0}^{2^{p-1}-1} a_{k m} w_{m}(y)+\sum_{m=2^{p-1}}^{2^{n+1}-1} a_{k m} w_{m}(y)\right| \\
& >C+\delta_{k p}+\cdots+\delta_{k n}-\delta_{k n+1}>C^{\prime}
\end{aligned}
$$

на $\Delta_{n+1}^{\prime}$ при $k \in\left\{i_{r}\right\}_{r} \geqslant N$. Значит, и в случае в) (26) выполняется для $j_{1}=n+1$. Таким образом, формула (26) доказана.

Пусть $\left\{C_{n}\right\}$ - убывающая последовательность чисел таких, что $\frac{C}{2}<C_{n}<C$ $(n=1,2,3, \ldots)$.

В силу (25) и (26) найдутся два непересекающихся интервала $\Delta^{0}$ и $\Delta^{1}$ ранга $j(1)>s$, на каждом из которых $\left|\sum_{m=0}^{2^{j(1)}-1} a_{k m} w_{m}(y)\right|>C_{1}$ для некоторого $k=N_{1}$. На каждом из интервалов $\Delta^{0}$ и $\Delta^{1}$ в силу (24)-(26) также найдутся по два непересекающихся интервала ранга $j(2)>j(1)\left(\Delta^{00}\right.$ и $\Delta^{01}$ на $\Delta^{0}$ и $\Delta^{10}$ и $\Delta^{11}$ на $\left.\Delta^{1}\right)$, на которых $\left|\sum_{m=0}^{2^{j(2)}-1} a_{k m} w_{m}(y)\right|>C_{2}$ для $k=N_{2}>N_{1}$.

Продолжая это построение далее, на $n$-м шаге определим с помощью (24)-(26) $2^{n}$ непересекающихся интервалов $\Delta^{\varepsilon_{1} \varepsilon_{2} \ldots \varepsilon_{n}}\left(\right.$ где $\left.\varepsilon_{i}=0,1 ; i=1,2, \ldots, n\right)$ ранга $j(n)>j(n-1)$ таких, что

$$
\Delta^{\varepsilon_{1} \ldots \varepsilon_{n-1} \varepsilon_{n}} \subset \Delta^{\varepsilon_{1} \ldots \varepsilon_{n-1}}, \quad \Delta^{\varepsilon_{1} \ldots \varepsilon_{n-1} 0} \cap \Delta^{\varepsilon_{1} \ldots \varepsilon_{n-1} 1}=\varnothing,
$$

на каждом из интервалов $\Delta^{\varepsilon_{1} \ldots \varepsilon_{n}}$

$$
\left|\sum_{m=0}^{2^{j(n)}-1} a_{k m} w_{m}(y)\right|>C_{n} \text { для } k=N_{n}>N_{n-1} \text {. }
$$

Пусть $\left(\varepsilon_{1}, \ldots, \varepsilon_{n}, \ldots\right)$ - последовательности из нулей и единиц. Из свойств интервалов $\Delta^{\varepsilon_{1} \ldots \varepsilon_{n}}$ следует, что найдется точка $t\left(\varepsilon_{1}, \ldots, \varepsilon_{n}, \ldots\right)$ такая, что

$$
\bigcap_{n=1}^{\infty} \overline{\Delta^{\varepsilon_{1} \ldots \varepsilon_{n}}}=\left\{t\left(\varepsilon_{1}, \ldots, \varepsilon_{n}, \ldots\right)\right\}
$$

где $\bar{\Delta}$ - замыкание $\Delta$.

Заметим, что для разных последовательностей $\left(\varepsilon_{1}, \ldots, \varepsilon_{n}, \ldots\right)$ числа $t\left(\varepsilon_{1}, \ldots\right.$, $\varepsilon_{n}, \ldots$ ) могут совпасть, только если в этих последовательностях все $\varepsilon_{i}$, начиная с некоторого, равны 0 , либо все $\varepsilon_{i}$, начиная с некоторого, равны 1 . Таких последовательностей счетное число. Следовательно, найдется континуум различных двоично-иррациональных и не принадлежащих множеству $B$ чисел $y=t\left(\varepsilon_{1}, \ldots, \varepsilon_{n}, \ldots\right)$. 
Но в таком случае для этих чисел и $\bigcap_{n=1}^{\infty} \Delta^{\varepsilon_{1} \ldots \varepsilon_{n}}=\left\{t\left(\varepsilon_{1}, \ldots, \varepsilon_{n}\right)\right\}$. Для таких чисел $y$ в силу (31) выполняются неравенства

$$
\left|\sum_{m=0}^{2^{j(n)}-1} a_{N_{n} m} w_{m}(y)\right|>\frac{C}{2} \quad(n=1,2,3, \ldots)
$$

что противоречит условию (19) теоремы. Следовательно, условие (20) выполняется и теорема доказана.

В. А. Скворцов и А. А. Талалян [10] доказали теорему единственности для кратных рядов Хаара, которая допускает переформулировку для кратных рядов Уолша, так как речь в теореме идет о частичных суммах с номерами $2^{k_{1}}, \ldots, 2^{k_{d}}$. Нам понадобится

СЛЕДСТВИЕ ТЕОРЕМЫ СКВОРцОВА-ТАЛАЛЯНА. Пусть для двойного ряда $S$ по системе Уолша выполняются условия

$$
\lim _{\min (N, M) \rightarrow \infty} S_{2^{N}, 2^{M}}(x, y) \leqslant f(x, y) \leqslant \varlimsup_{\min (N, M) \rightarrow \infty} S_{2^{N}, 2^{M}}(x, y)
$$

всюду на $[0,1)^{2}$, за исключением, быть может, счетного множества, где $f(x, y)-$ конечная, суммируемая на $[0,1)^{2}$ функиия, и

$$
\lim _{\min (N, M) \rightarrow \infty} \frac{1}{2^{N+M}} S_{2^{N}, 2^{M}}(x, y)=0 \quad \text { для всех } \quad(x, y) \in[0,1)^{2} .
$$

Тогда $S$ является рядом Фурье-Уолиа функиии $f(x, y)$.

Приведенным ядром ряда $S(7)$ называется множество точек, где он неограниченно расходится, т.е. множество

$$
\mathscr{N}=\left\{(x, y): \varlimsup_{\min (N, M) \rightarrow \infty}\left|S_{N M}(x, y)\right|=\infty\right\}
$$

Ряд $S$ называется нуль-рядом, если он почти всюду сходится к нулю, но не все его коэффициенты равны нулю.

В качестве примера нуль-ряда класса $\mathscr{K}_{2}$ можно взять ряд, являющийся формальным произведением какого-нибудь одномерного нуль-ряда $\sum_{n=0}^{\infty} a_{n} w_{n}(x)$ на полином $w_{0}(y)+w_{1}(y)$.

Покажем, что приведенное ядро нуль-ряда класса $\mathscr{K}_{2}$ несчетно и не первой категории на себе, аналогично соответствующему свойству одномерных нуль-рядов.

ЛЕмма 4. Пусть $S$ - нуль-ряд класса $\mathscr{K}_{2}, \mathscr{E}-$ ядро и $\mathcal{N}-$ приведенное ядро $S$. Если $\mathscr{E} \cap G \neq \varnothing$ для некоторого открытого множества $G$, то мнохсество $\mathcal{N} \cap G$ несчетно. 
Доказательство. Пусть $\left(x_{0}, y_{0}\right) \in \mathscr{E} \cap G, \Pi \in \mathscr{P}_{\text {и }}\left(x_{0}, y_{0}\right) \in \Pi \subset G$. Рассмотрим формальное произведение $\widetilde{S}$ ряда $S$ на полином $\chi_{\Pi}(x, y)$. В силу теоремы $1 \widetilde{S}-$ нуль-ряд класса $\mathscr{K}_{2}$ с ядром $\mathscr{E} \cap \Pi$ и приведенным ядром $\mathscr{N} \cap$ П. Если $\mathscr{N} \cap G$ счетно, то частичные суммы ряда $\widetilde{S}$ ограничены в каждой точке $[0,1)^{2}$, за исключением, быть может, счетного множества. Так как $\widetilde{S}$ - класса $\mathscr{K}_{2}$, то в силу теоремы 6 его коэффициенты $\widetilde{a}_{k m} \rightarrow 0$ при $k+m \rightarrow \infty$. Следовательно, выполняется условие

$$
\left|\frac{1}{2^{N+M}} \widetilde{S}_{2^{N}, 2^{M}}(x, y)\right| \leqslant \frac{1}{2^{N+M}} \sum_{k=0}^{2^{N}-1} \sum_{m=0}^{2^{M}-1}\left|\widetilde{a}_{k m}\right| \rightarrow 0,
$$

если $\min (N, M) \rightarrow+\infty$. Тогда в силу следствия из теоремы Скворцова-Талаляна ряд $\widetilde{S}$ является рядом Фурье своей суммы, т.е. $\widetilde{S} \equiv 0$. Это противоречит тому, что $\widetilde{S}$ - нуль-ряд. Следовательно, множество $\mathscr{N} \cap G$ несчетно. Лемма доказана.

ЛЕмма 5. Пусть $S$ - нуль-ряд класса $\mathscr{K}_{2}, \mathscr{E}$ - ядро и $\mathscr{N}$ - приведенное ядро этого нуль-ряда; $L$-мноэсество, состоящее из конечного числа вертикальньхх $x=c_{i}\left(c_{i} \in Q_{2}\right)$ и горизонтальных отрезков $y=d_{i}\left(d_{i} \in Q_{2}\right)$ на $[0,1)^{2}$; $\Pi \in \mathscr{P}$ - прямоугольник такой, что $\mathscr{N} \cap \Pi \neq \varnothing, k \in \mathbb{N}$. Тогда найдутся два прямоугольника $\Pi_{0}$ и $\Pi_{1}$ из $\mathscr{P}$ такие, что $\bar{\Pi}_{0} \cap \bar{\Pi}_{1}=\varnothing, \Pi_{i} \subset \Pi, \bar{\Pi}_{i} \cap L=\varnothing$, $\mathscr{N} \cap \Pi_{i} \neq \varnothing(i=0,1) u$

$$
\left|S_{N_{i} M_{i}}(x, y)\right|>k
$$

для $(x, y) \in \Pi_{i}$ и некоторых $N_{i}>k, M_{i}>k(i=0,1)$.

ДокАЗАТЕЛЬство. Пусть Г - граница прямоугольника П. Покажем, что найдется точка $\left(x_{0}, y_{0}\right) \in(\mathscr{N} \cap \Pi) \backslash(\Gamma \cup L)$. Рассмотрим формальное произведение ряда $S$ на полином $\chi_{\Pi}(x, y)-$ ряд $\widetilde{S}$. В силу теоремы 1 это тоже нуль-ряд класса $\mathscr{K}_{2}$ с ядром $\mathscr{E} \cap \Pi$ и приведенньм ядром $\mathscr{N} \cap \Pi$. Допустим, что $\mathscr{N} \cap \Pi \subset \Gamma \cup L$. Тогда в силу леммы $4 \mathscr{E} \cap \Pi \subset \Gamma \cup L$. Но множество $\Gamma \cup L$ является $U$-множеством в силу теоремы С Лукомского. Следовательно, $\widetilde{S}$ сходится к нулю всюду вне $U$-множества и все его коэффициенты равны нулю. Это противоречит тому, что $\widetilde{S}-$ нуль-ряд. Следовательно, $(\mathscr{N} \cap \Pi) \backslash(\Gamma \cup L) \neq \varnothing$.

Пусть $\left(x_{0}, y_{0}\right) \in(\mathscr{N} \cap \Pi) \backslash(\Gamma \cup L)$. Тогда найдется окрестность точки $\left(x_{0}, y_{0}\right)$, не пересекаюшаяся с $\Gamma \cup L$, и в силу леммы 4 найдется другая точка $\left(x_{1}, y_{1}\right) \in$ $(\mathscr{N} \cap \Pi) \backslash(\Gamma \cup L)$. Так как $\left(x_{i}, y_{i}\right)(i=0,1)$ - точки приведенного ядра, то найдутся номера $N_{i}, M_{i}>k$ такие, что

$$
\left|S_{N_{i} M_{i}}\left(x_{i}, y_{i}\right)\right|>k \quad(i=0,1) .
$$

Так как частичные суммы $S_{N_{i} M_{i}}$ постоянны на прямоугольниках, являющихся прямыми произведениями двоичных интервалов рангов $N_{i}$ и $M_{i}$, то можно выбрать такие прямоугольники $\Pi_{i} \in \mathscr{P}(i=0,1)$, что выполняются все условия леммы. Лемма доказана.

ТеОРема 7. Пусть $S$ - нуль-ряд класса $\mathscr{K}_{2}, \mathscr{E}$ - ядро и $\mathscr{N}$ - приведенное ядро этого нуль-ряда; $J$ - обгединение всех вертикальных и горизонтальных отрезков вида $x=r, r \in Q_{2}$, и $y=r, r \in Q_{2}$, из $[0,1)^{2}$. Тогда множество $\mathscr{N} \backslash J$ несчетно и $\mathscr{N}$ не первой категории на себе. 
ДокАЗАТЕЛЬСтво. Множество $\mathscr{N}$ можно представить в виде

$$
\mathscr{N}=\bigcap_{k=1}^{\infty} \bigcup_{N=k}^{\infty} \bigcup_{M=k}^{\infty}\left\{(x, y):\left|S_{N M}(x, y)\right|>k\right\}
$$

Функция $S_{N} M(x, y)$ непрерывна всюду на $[0,1)^{2}$, за исключением, быть может, замкнутого множества $I_{N M}$, содержашегося в объединении конечного числа вертикальных и горизонтальных отрезков, $I_{N M} \subset J$. Положим

$$
A_{k N M}=\left\{(x, y):\left|S_{N M}(x, y)\right|>k\right\} \text {. }
$$

Так как на $[0,1)^{2} \backslash I_{N M}$ функция $S_{N M}(x, y)$ непрерывна, то $V_{k N M}=A_{k N M} \backslash I_{N M}$ - открытое множество. Введем обозначение $Z_{k N M}=A_{k N M} \cap I_{N M}$. Следовательно, $A_{k N M}=V_{k N M} \cup Z_{k N M}$, где $V_{k N M}$ - открытое подмножество $[0,1)^{2}$, $Z_{k N M} \subset J$ и $V_{k N M} \cap Z_{k N M}=\varnothing$. Положим $V_{k}=\bigcup_{N=k}^{\infty} \bigcup_{M=k}^{\infty} V_{k N M}$, тогда $V_{k}-$ открытое множество и в силу (32)

$$
\bigcap_{k=1}^{\infty} V_{k} \subset \mathscr{N} \subset \bigcap_{k=1}^{\infty} V_{k} \cup J .
$$

Представим множество $J$ в виде $J=\bigcup_{n=1}^{\infty} L_{n}$, где каждое множество $L_{n}$ является объединением конечного числа вертикальных и горизонтальных отрезков. Пусть $\Pi=[0,1)^{2}, k=1, L=L_{1}$. С помощью леммы 5 определим прямоугольники $\Pi_{0}$ и $\Pi_{1}$ с указанными в лемме свойствами. На $n$-м шаге с помошью леммы 5 построим $2^{n}$ прямоугольников $\Pi_{\varepsilon_{1} \ldots \varepsilon_{n}} \in \mathscr{P}\left(\varepsilon_{i}=0,1\right)$ со свойствами

$$
\begin{gathered}
\Pi_{\varepsilon_{1} \ldots \varepsilon_{n}} \subset \Pi_{\varepsilon_{1} \ldots \varepsilon_{n-1}}, \quad \bar{\Pi}_{\varepsilon_{1} \ldots \varepsilon_{n-1} 0} \cap \bar{\Pi}_{\varepsilon_{1} \ldots \varepsilon_{n-1} 1}=\varnothing, \\
\left|S_{N M}(x, y)\right|>n \quad \text { для } \quad(x, y) \in \Pi_{\varepsilon_{1} \ldots \varepsilon_{n}} \text { и для некоторых } \quad M, N>n, \\
\bar{\Pi}_{\varepsilon_{1} \ldots \varepsilon_{n}} \cap L_{n}=\varnothing .
\end{gathered}
$$

Можно считать, что с ростом $n$ диаметры прямоугольников $\Pi_{\varepsilon_{1} \ldots \varepsilon_{n}}$ стремятся к нулю (уменьшая, если нужно, их диаметр на каждом шаге). Тогда каждой последовательности $\left(\varepsilon_{1}, \varepsilon_{2}, \ldots, \varepsilon_{n}, \ldots\right)$ из нулей и единиц соответствует точка $\{(x, y)\}=\bigcap_{n=1}^{\infty} \bar{\Pi}_{\varepsilon_{1} \ldots \varepsilon_{n}}$. Из (33) следует, что разным последовательностям $\left(\varepsilon_{1}, \varepsilon_{2}, \ldots, \varepsilon_{n}, \ldots\right)$ соответствуют разные точки $(x, y)$, и таких точек - континуум, причем $(x, y) \notin J$. Следовательно, обе координаты таких точек $(x, y)$ двоично-иррациональные. Отсюда следует, что $\{(x, y)\}=\bigcap_{n=1}^{\infty} \Pi_{\varepsilon_{1} \ldots \varepsilon_{n}}$ и, следовательно, $(x, y) \in \mathscr{N} \backslash J$. Таким образом, множество $\mathcal{N} \backslash J$ имеет мощность континуума и $V=\bigcap_{k=1}^{\infty} V_{k}$ - континуальное $G_{\delta}$-множество.

Покажем, что $\mathscr{N} \subset \bar{V}$. Так как $\mathscr{N} \backslash J \subset V$, то достаточно показать, что $\mathscr{N} \cap J \subset \bar{V}$. Допустим, что $(\mathscr{N} \cap J) \backslash \bar{V} \neq \varnothing$. Положим $G=[0,1)^{2} \backslash \bar{V}$. Тогда найдется прямоугольник $\Pi \in \mathscr{P}$ такой, что $\Pi \subset G, \Pi \cap \mathcal{N} \cap J \neq \varnothing$. Рассмотрим ряд $\widetilde{S}$, являющийся формальным произведением $S$ на полином $\chi_{\Pi}(x, y)$. В силу теоремы 1 это - нуль-ряд класса $\mathscr{K}_{2}$ с приведенным ядром $\mathscr{N}_{1}=\Pi \cap \mathcal{N} \cap J \subset J$. Но это противоречит только что доказанному, тому, что $\mathscr{N}_{1} \backslash J \neq \varnothing$. Таким образом $\mathscr{N} \subset \bar{V}$. 
Если $\mathscr{N}$ первой категории на себе, то $\mathscr{N}=\bigcup_{k=1}^{\infty} A_{k}$, где $A_{k}$ нигде не плотны в $\mathscr{N}$.

Тогда $V=V \cap \mathscr{N}=\bigcup_{k=1}^{\infty}\left(V \cap A_{k}\right)$.

Так как $V$ - непустое $G_{\delta}$-множество, то $V$ не первой категории на себе (см., например, $\left[11 ; \S 34\right.$, пा. IV, V]). Следовательно, найдется номер $k_{0}$ и открытое множество $G_{1}$ такие, что $V \cap A_{k_{0}}$ плотно в $G_{1} \cap V \neq \varnothing$. Так как $V \cap A_{k_{0}}$ нигде не плотно в $\mathscr{N}$, то найдется открытое множество $G_{2} \subset G_{1}$ такое, что $\mathscr{N} \cap G_{2} \neq \varnothing$, $G_{2} \cap V \cap A_{k_{0}}=\varnothing$. Тогда и $G_{2} \cap V=\varnothing$, так как $V \cap A_{k_{0}}$ плотно в $G_{1} \cap V$. Но это противоречит тому, что $\mathscr{N} \subset \bar{V}$. Следовательно, $\mathscr{N}$ не первой категории на себе. Теорема доказана.

Из теоремы 7 и леммы 4 следует, что семейство $M\left(\mathscr{K}_{2}\right)$-множеств меры нуль является семейством со свойством приведенного ядра. Следовательно, в силу теоремы Е справедлива

Теорема 8. Пусть $E_{k}-U\left(\mathscr{K}_{2}\right)$-множества меры нуль, замкнутые относительно $E=\bigcup_{k=1}^{\infty} E_{k}$. Тогда $E-U\left(\mathscr{K}_{2}\right)$-множество.

Из теоремы 8 и леммы 2 следует результат для обычных $U$-множеств.

Теорема 9. Пусть $E_{k}-U$-мнолсества меры нуль для двойных рядов Уолша, замкнутые относительно $E=\bigcup_{k=1}^{\infty} E_{k}$. Если $[0,1)^{2} \backslash E$ содержит какое-нибудь существенное множество, то Е является $U$-множеством.

Замечание. Если в теореме 9 потребовать, что $E=\bigcup_{k=1}^{\infty} E_{k}$ замкнуто или хотя бы типа $G_{\delta}$, то теорема 9 справедлива без дополнительного требования - меpa $E_{k}$ равна нулю.

Теоремы 6-9 также переносятся на кратный случай. Во введении сформулирована теорема $9^{\prime}$ для $d$-кратного случая и сушественного множества, состояшего из объединения поверхностей $x_{i}=0(i=1, \ldots, d)$.

Приведем пример $U$-множества, полученного с помощью теоремы 9 . Пусть $A-$ совершенное $U$-множество на $[0,1), 0 \notin A$. Тогда множество $(A \times(0,1)) \cup((0,1) \times A)$ не содержит сушественное множество, $x=0$ и $y=0$, и является в силу теоремы 9 $U$-множеством.

Один из фундаментальных результатов теории единственности однократных рядов состоит в том, что множество положительной меры является $M$-множеством и для тригонометрических рядов, и для рядов Уолша. В кратном случае это - открытый как для рядов Уолша, так и для тригонометрических рядов

Вопрос. Является ли множество положительной меры обязательно $M$-множеством?

\section{§3. Тригонометрический случай}

Обратимся теперь к тригонометрическому случаю.

Обозначим через $\mathscr{K}_{2}(T)$ класс двойных тригонометрических рядов

$$
\sum_{m, n=-\infty}^{\infty} c_{m n} e^{i m x} e^{i n y}, \quad c_{-m,-n}=\overline{c_{m n}}
$$


таких, что

$$
\begin{aligned}
\lim _{\min (|M|, N) \rightarrow \infty} \sum_{|n| \leqslant N} c_{M n} e^{i n y}=0, & y \in[0,2 \pi), \\
\lim _{\min (M,|N|) \rightarrow \infty} \sum_{|m| \leqslant M} c_{m N} e^{i m x}=0, & x \in[0,2 \pi), \\
\sup _{M, N}\left|\sum_{|n| \leqslant N} c_{M n} e^{i n y}\right| \leqslant C(y)<\infty, & y \in[0,2 \pi), \\
\sup _{M, N}\left|\sum_{|m| \leqslant M} c_{m N} e^{i m x}\right| \leqslant C(x)<\infty, & x \in[0,2 \pi) .
\end{aligned}
$$

Отметим, что из условий (35), (36) не следуют, вообще говоря, условия $(37),(38)$. Действительно, рассмотрим тригонометрический ряд (34), у которого $c_{00}=1$, $c_{0 n}=\frac{1}{|n|}$, если $n \neq 0, c_{m n}=0$, если $m \neq 0$. Тогда $\sum_{|n| \leqslant N} c_{M n} e^{i n y} \equiv 0$ при $M \neq 0$ и $\sum_{|m| \leqslant M} c_{m N} e^{i m x}=\frac{1}{|N|}$ при $N \neq 0$, следовательно, условия (35) и (36) вьполняются. Но при $m=0, y=0$ имеем $\sum_{|n| \leqslant N} c_{0 n} e^{i n 0}=1+2 \sum_{n=1}^{N} \frac{1}{n} \rightarrow \infty$ при $N \rightarrow \infty$; т.е. не выполняется условие $(37)$.

Укажем некоторые достаточные условия принадлежности ряда классу $\mathscr{K}_{2}(T)$.

Напомним, что множество $E \subset[0,2 \pi)$ называется $R$-множсеством, если сушествует тригонометрический ряд

$$
\frac{a_{0}}{2}+\sum_{n=1}^{\infty} a_{n} \cos n x+b_{n} \sin n x
$$

которьй сходится на $E$, а коэффициенты его не стремятся к нулю.

Множество $E \subset[0,2 \pi)$ назьвается множеством Арбо, кратко, Ar-множеством, если существует возрастающая последовательность натуральных чисел $\left\{n_{k}\right\}$ такая, что $\lim _{k \rightarrow \infty} \sin n_{k} x=0, x \in E$.

Известно (см. $[12$; гл. XIV,$\S 23])$, что $R$-множества являются множествами меры 0 и первой категории, счетные множества являются $R$-множествами, а, например, канторовские множества с постоянньм отношением разбиения не являются $R$-множествами.

Долгое время вопрос о взаимоотношении классов $R$ - и Ar-множеств был открыт. В 1992 г. С. Кахан [13] доказал, что всякое $R$-множество является Ar-множеством, a C.В. Конягин [14] - что всякое Ar-множество является $R$-множеством.

Назовем множество $A \subset[0,2 \pi)^{2}$ существенным для двойных тригонометрических рядов, если для каждого $x \in[0,2 \pi)$ и каждого $y \in[0,2 \pi)$ множества

$$
A_{x}=\{y:(x, y) \in A\} \quad \text { и } E_{y}=\{x:(x, y) \in A\}
$$

не являются $R$-множествами. 
Лемма 6. Пусть $A_{m n}, B_{m n} \in \mathbb{R}(m, n=0,1,2, \ldots), E \subset[0,2 \pi), E$ не является $R$-мнохеством. Если

$$
\sup _{m, n}\left|A_{m n} \cos m x+B_{m n} \sin m x\right| \leqslant C_{1}=\text { const, } \quad x \in E
$$

то найдется константа $C$ такая, что

$$
\left|A_{m n}\right| \leqslant C(m, n=0,1,2, \ldots), \quad\left|B_{m n}\right| \leqslant C(n=0, \quad m, n=1,2,3, \ldots) .
$$

Eсли

$$
\lim _{\min (m, n) \rightarrow \infty}\left(A_{m n} \cos m x+B_{m n} \sin m x\right)=0, \quad x \in E,
$$

mo

$$
\lim _{\min (m, n) \rightarrow \infty} A_{m n}=\lim _{\min (m, n) \rightarrow \infty} B_{m n}=0 .
$$

ДокАЗАТЕЛьство. При $m=0$ из (39) следует, что $\left|A_{0 n}\right| \leqslant C_{1}(n=0,1,2, \ldots)$. Далее рассматриваем $m \geqslant 1$. Положим $\rho_{m n}=\sqrt{A_{m n}^{2}+B_{m n}^{2}}$. Тогда

$$
A_{m n} \cos m x+B_{m n} \sin m x=\rho_{m n} \sin \left(m x+\varphi_{m n}\right), \quad \text { где } \varphi_{m n} \in[0,2 \pi) .
$$

Если (40) не выполняется, то найдутся номера $m_{k}, n_{k}(k \in \mathbb{N})$ такие, что

$$
\lim _{k \rightarrow \infty} \rho_{m_{k} n_{k}}=\infty, \quad \lim _{k \rightarrow \infty} \varphi_{m_{k} n_{k}}=\varphi
$$

Отсюда и из (39) следует, что

$$
\lim _{k \rightarrow \infty} \sin \left(m_{k} x+\varphi\right)=0, \quad x \in E .
$$

Если $m_{k}=l \neq 0$ для бесконечного числа номеров $k$, то последнее равенство невозможно, так как $E$ - бесконечное множество. Значит, $\lim _{k \rightarrow \infty} m_{k}=\infty$. Выберем такую последовательность номеров $k_{r}$, что последовательность $\nu_{r}=m_{k_{r+1}}-m_{k_{r}}$ возрастает. Тогда

$$
\lim _{r \rightarrow \infty} \sin \nu_{r} x=\lim _{r \rightarrow \infty} \sin \left(\left(m_{k_{r+1}} x+\varphi\right)-\left(m_{k_{r}} x+\varphi\right)\right)=0, \quad x \in E .
$$

Но это противоречит тому, что $E$ не является $R$-множеством. Следовательно, выполняется условие (40). То, что из (41) следует (42), доказывается аналогично (доказательство есть в [15]). Лемма доказана.

Из леммы 6 легко следует

ЛЕмма 7. Из условия (35) (а также из (36)) следует, что

$$
\lim _{\min (|m|,|n|) \rightarrow \infty} c_{m n}=0
$$

Из условия (37) (а также из (38)) следует, что

$$
\left|c_{m n}\right| \leqslant C=\mathrm{const} \quad(m, n \in \mathbb{Z})
$$


ДокАЗАТЕЛЬСТво. Пусть $c_{m n}=a_{m n}+i b_{m n}, a_{m n}, b_{m n} \in \mathbb{R}$. Тогда

$$
\begin{aligned}
& \sum_{|n| \leqslant N} c_{M n} e^{i n y}-\sum_{|n| \leqslant N-1} c_{M n} e^{i n y}=\sum_{|n|=N} c_{M n} e^{i n y} \\
& =\left(a_{M N}+a_{M,-N}\right) \cos N y+\left(b_{M,-N}-b_{M N}\right) \sin N y \\
& \quad+i\left[\left(b_{M N}+b_{M,-N}\right) \cos N y+\left(a_{M N}-a_{M,-N}\right) \sin N y\right] .
\end{aligned}
$$

Так как $[0,2 \pi)$ не является $R$-множеством, то отсюда, из $(35)$ и леммы 6 следует сходимость к нулю коэффициентов этого выражения $a_{M N} \pm a_{M,-N}, b_{M,-N} \pm b_{M N}$ при $\min (M, N) \rightarrow \infty$ и выполнение условия (43). Аналогично, из условия (37) и леммы 6 следует (44). (Рассматривая $\sum_{|m| \leqslant M} c_{m N} e^{i m x}-\sum_{|m| \leqslant M-1} c_{m N} e^{i m x}$, придем к доказательству (43) и (44) из (36) и (38) соответственно.) Лемма доказана.

ЛЕмма 8. Если двойной тригонометрический ряд (34) сходится (по прямоугольникам) на некотором существенном множестве $A$, то для этого ряда выполняются условия (35) и (36). Если частичные суммы ряда (34) ограничены на $A$, то выполняются условия (37) и (38).

ДокаЗАтЕльство. Пусть $S_{M N}(x, y)=\sum_{|m| \leqslant M} \sum_{|n| \leqslant N} c_{m n} e^{i m x} e^{i n y}$. Из сходимости ряда на множестве $A$ следует, что

$$
\lim _{\min (M, N) \rightarrow \infty}\left(S_{M N}(x, y)-S_{M-1, N}(x, y)\right)=0, \quad(x, y) \in A .
$$

При этом

$$
\begin{aligned}
S_{M N}(x, y)-S_{M-1, N}(x, y) & =\sum_{|m|=M} \sum_{|n| \leqslant N} c_{m n} e^{i m x} e^{i n y} \\
& =2 \operatorname{Re}\left(e^{i M x} \sum_{|n| \leqslant N} c_{M n} e^{i n y}\right)
\end{aligned}
$$

Зафикисируем $y \in[0,2 \pi)$ и обозначим

$$
\sum_{|n| \leqslant N} c_{M n} e^{i n y}=\alpha_{M N}+i \beta_{M N}, \quad \alpha_{M N}, \beta_{M N} \in \mathbb{R}, \quad M>0 .
$$

В силу предыдущего

$$
\lim _{\min (M, N) \rightarrow \infty} \operatorname{Re}\left((\cos M x+i \sin M x)\left(\alpha_{M N}+i \beta_{M N}\right)\right)=0, \quad x \in E_{y},
$$

т.e.

$$
\lim _{\min (M, N) \rightarrow \infty}\left(\alpha_{M N} \cos M x-\beta_{M N} \sin M x\right)=0, \quad x \in E_{y} .
$$

Так как $E_{y}$ не $R$-множество (в силу того, что $A$ - сушественное множество), то отсюда и из леммы 6 следует, что

$$
\lim _{\min (M, N) \rightarrow \infty} \alpha_{M N}=\lim _{\min (M, N) \rightarrow \infty} \beta_{M N}=0 .
$$


Таким образом,

$$
\lim _{\min (M, N) \rightarrow \infty} \sum_{|n| \leqslant N} c_{M n} e^{i n y}=0, \quad y \in[0,2 \pi), \quad M>0 .
$$

Так как для $M>0$ имеем $\sum_{|n| \leqslant N} c_{-M n} e^{i n y}=\overline{\sum_{|n| \leqslant N} c_{M n} e^{i n y}}$, то (35) выполняется. Из ограниченности $S_{M N}(x, y)$ на $A$ подобньм же образом следует (37). Аналогично доказываются формулы (36), (38). Лемма доказана.

Аналогично одномерному случаю, формальным произведением ряда $S(34)$ на ряд $\sum_{m=-\infty}^{\infty} a_{m} e^{i m x}$ называется ряд

$$
\sum_{m, n=-\infty}^{\infty} K_{m n} e^{i m x} e^{i n y}, \quad K_{m n}=\sum_{\nu=-\infty}^{\infty} c_{\nu n} a_{m-\nu}
$$

в предположении, что ряды, определяющие $K_{m n}$, сходятся.

Подобно лемме Райхмана для однократного тригонометрического ряда (см. [12; гл. I, $\S 71$, лемма 1]) доказьвается

ЛЕмма 9. Пусть коэффициенты ряда $S$ (34) удовлетворяют условиям $\left|c_{m n}\right|<C u \lim _{\min (|m|,|n|) \rightarrow \infty} c_{m n}=0 u \sum_{m=-\infty}^{\infty}\left|a_{m}\right|<\infty$. Тогда для коэффициентов формального произведения ряда $\bar{S}$ на $\lambda_{1}(x)=\sum_{m=-\infty}^{\infty} a_{m} e^{i m x}-$ ряда (45) - выполняются условия

$$
\left|K_{m n}\right|<\text { const, } \quad \lim _{\min (|m|,|n|) \rightarrow \infty} K_{m n}=0 .
$$

TEOPЕмA 10. Пусть $S$ - ряд класса $\mathscr{K}_{2}(T)$, функция $\lambda_{1}(x)=\sum_{\nu=-\infty}^{\infty} a_{\nu} e^{i \nu x}$ задается абсолютно сходящимся тригонометрическим рядом, причем

$$
\sum_{\nu=-\infty}^{\infty}\left|\nu a_{\nu}\right|<\infty
$$

Тогда формальное произведение ряда $S$ на ряд Фурье функции $\lambda_{1}(x)$ - ряд $\sigma$ равносходится с рядом $\lambda_{1}(x) \cdot S$, т.е. разность частичных сумм $\sigma_{M N}(x, y)-$ $\lambda_{1}(x) S_{M N}(x, y)$ сходится $\kappa$ нулю при $\min (M, N) \rightarrow \infty$ для $(x, y) \in[0,2 \pi)^{2}$. Ряд $\sigma$ тоже класса $\mathscr{K}_{2}(T)$. Аналогично, если $\lambda_{2}(y)=\sum_{\nu=-\infty}^{\infty} b_{\nu} e^{i \nu y}$,

$$
\sum_{\nu=-\infty}^{\infty}\left|\nu b_{\nu}\right|<\infty
$$

то формальное произведение ряда $S$ на $\lambda_{2}(y)$ равносходится с рядом $\lambda_{2}(y) \cdot S$ и является рядом класса $\mathscr{K}_{2}(T)$. 
ДОКАЗАТЕЛЬСТВО. ПоЛоЖим

$$
a_{\nu}^{*}= \begin{cases}a_{0}-\lambda_{1}(x), & \nu=0 \\ a_{\nu}, & \nu \neq 0\end{cases}
$$

Тогда $\sum_{\nu=-\infty}^{\infty} a_{\nu}^{*} e^{i \nu x}=0$. Рассмотрим ряд

$$
S^{*}=\sum_{m, n}\left(\sum_{\nu} c_{\nu n} a_{m-\nu}^{*}\right) e^{i m x} e^{i n y}
$$

Из определения ряда $S^{*}$ следует, что частичные суммы рядов $S^{*}, S$ и $\sigma$ связаны равенством

$$
S_{M N}^{*}=\sigma_{M N}-\lambda_{1}(x) S_{M N} .
$$

Покажем, что ряд $S^{*}$ всюду на $[0,2 \pi)^{2}$ сходится к нулю.

Положим $R_{k}(x)=\sum_{\nu=k}^{\infty} a_{\nu}^{*} e^{i \nu x}$. Для каждого натурального $k$

$$
\begin{aligned}
R_{-k}(x) & =\sum_{\nu=-k}^{\infty} a_{\nu}^{*} e^{i \nu x}=\sum_{\nu=-k}^{\infty} a_{\nu}^{*} e^{i \nu x}-\sum_{\nu=-\infty}^{\infty} a_{\nu}^{*} e^{i \nu x} \\
& =-\sum_{\nu=-\infty}^{-(k+1)} a_{\nu} e^{i \nu x}=-\sum_{\nu=k+1}^{\infty} a_{-\nu} e^{-i \nu x}=-\sum_{\nu=k+1}^{\infty} \overline{a_{\nu} e^{i \nu x}}=-\overline{R_{k+1}}(x) .
\end{aligned}
$$

Так как

$$
\sum_{k=0}^{\infty}\left|R_{k}(x)\right| \leqslant \sum_{k=0}^{\infty} \sum_{\nu=k}^{\infty}\left|a_{\nu}^{*}\right| \leqslant 2 \sum_{\nu=-\infty}^{\infty}\left|a_{\nu}\right|+\sum_{k=1}^{\infty} k\left|a_{k}\right|<\infty
$$

то и ряд $\sum_{k=-\infty}^{\infty}\left|R_{k}(x)\right|$ сходится.

Преобразуем теперь

$$
\begin{aligned}
S_{M N}^{*} & =\sum_{|m| \leqslant M} \sum_{|n| \leqslant N}\left(\sum_{\nu=-\infty}^{\infty} c_{\nu n} a_{m-\nu}^{*}\right) e^{i m x} e^{i n y} \\
& =\sum_{\nu=-\infty}^{\infty} \sum_{|n| \leqslant N} c_{\nu n} e^{i n y} \sum_{|m| \leqslant M} a_{m-\nu}^{*} e^{i m x} \\
& =\sum_{\nu=-\infty}^{\infty} \sum_{|n| \leqslant N} c_{\nu n} e^{i n y} \sum_{p=-M-\nu}^{M-\nu} a_{p}^{*} e^{i p x} e^{i \nu x} \\
& =\sum_{\nu=-\infty}^{\infty} e^{i \nu x} \sum_{|n| \leqslant N} c_{\nu n} e^{i n y}\left(R_{-M-\nu}(x)-R_{M-\nu+1}(x)\right) .
\end{aligned}
$$

Заметим далее, что

$$
\sum_{\nu=-\infty}^{\infty}\left|R_{-M-\nu}(x)-R_{M-\nu+1}(x)\right| \leqslant 2 \sum_{\nu=-\infty}^{\infty}\left|R_{\nu}(x)\right|<\infty .
$$


По лемме 7 (рассматривая в качестве $c_{m n}$ последовательность $\sum_{|n| \leqslant N} c_{m n} e^{i n y}$ и в качестве $\sum_{m=-\infty}^{\infty}\left|a_{m}\right|$ ряд $\left.\sum_{m=-\infty}^{\infty}\left|R_{-M-m}(x)-R_{M-m+1}(x)\right|\right)$, получаем

$$
\lim _{\min (M, N) \rightarrow \infty} S_{M N}^{*}(x, y)=0, \quad(x, y) \in[0,2 \pi)^{2} .
$$

В силу формулы (46) ряды $\sigma$ и $\lambda_{1}(x) \cdot S$ равносходятся на $[0,2 \pi)^{2}$. Покажем, что $\sigma$ - ряд класса $\mathscr{K}_{2}(T)$. Рассмотрим сначала

$$
\sum_{|n| \leqslant N} K_{M n} e^{i n y}=\sum_{|n| \leqslant N} \sum_{\nu=-\infty}^{\infty} a_{\nu} c_{M-\nu, n} e^{i n y}=\sum_{\nu=-\infty}^{\infty} a_{\nu} \sum_{|n| \leqslant N} c_{M-\nu, n} e^{i n y} .
$$

Свойства (35) и (37) для ряда $\sigma$ следуют из свойств (35) и (37) для ряда $S$ и леммы 7, где в качестве $c_{m n}$ берется $\sum_{|k| \leqslant n} c_{m k} e^{i k y}$. Рассмотрим теперь

$$
\begin{aligned}
\sum_{|m| \leqslant M} K_{m N} e^{i m x} & =\sum_{|m| \leqslant M} \sum_{\nu=-\infty}^{\infty} a_{\nu} c_{m-\nu, N} e^{i m x} \\
& =\sum_{\nu=-\infty}^{\infty} a_{\nu}\left(\sum_{|m| \leqslant M} c_{m-\nu, N} e^{i(m-\nu) x}\right) e^{i \nu x} \\
& =\sum_{\nu=-\infty}^{\infty} a_{\nu} e^{i \nu x} \sum_{k=-M-\nu}^{M-\nu} c_{k N} e^{i k x} \\
& =\sum_{\nu=-\infty}^{\infty} a_{\nu} e^{i \nu x} \sum_{|k| \leqslant M-\nu} c_{k N} e^{i k x} \\
& -\sum_{\nu=-\infty}^{\infty} a_{\nu} e^{i \nu x} \sum_{k=-M-\nu}^{-M+\nu-1} c_{k N} e^{i k x}
\end{aligned}
$$

В силу леммы $6\left|c_{k N}\right| \leqslant C=$ const. Учитывая еще свойство (38), получим

$$
\left|\sum_{|m| \leqslant M} K_{m N} e^{i m x}\right| \leqslant \sum_{\nu=-\infty}^{\infty}\left|a_{\nu}\right| \cdot C(x)+\sum_{\nu=-\infty}^{\infty} 2\left|\nu a_{\nu}\right| \cdot C
$$

т.е. для ряда $\sigma$ тоже выполняется свойство (38). Из свойства (36) для ряда $S$ и леммы 7, если в ней в качестве $c_{m n}$ взять $\sum_{|k| \leqslant m} c_{k n} e^{i k x}$, следует, что

$$
\lim _{\min (M, N) \rightarrow \infty} \sum_{\nu=-\infty}^{\infty} a_{\nu} e^{i \nu x} \sum_{|k| \leqslant M-\nu} c_{k N} e^{i k x}=0
$$

Заметим далее, что

$$
\begin{aligned}
& \left|\sum_{\nu=-\infty}^{\infty} a_{\nu} e^{i \nu x} \sum_{k=-M-\nu}^{-M+\nu-1} c_{k N} e^{i k x}\right| \\
& \leqslant \sum_{|\nu| \leqslant \frac{M}{2}} 2\left|\nu a_{\nu}\right| \cdot \max _{-\frac{3}{2} M \leqslant k \leqslant-\frac{M}{2}}\left|c_{k N}\right|+\sum_{|\nu|>\frac{M}{2}} 2\left|\nu a_{\nu}\right| \cdot C .
\end{aligned}
$$


Отсюда, из свойства (36) для ряда $S$ и сходимости ряда $\sum_{\nu=-\infty}^{\infty}\left|\nu a_{\nu}\right|$ вытекает, что

$$
\lim _{\min (M,|N|) \rightarrow \infty} \sum_{\nu=-\infty}^{\infty} a_{\nu} e^{i \nu x} \sum_{k=-M-\nu}^{-M+\nu-1} c_{k N} e^{i k x}=0
$$

Отсюда следует, что для ряда $\sigma$ выполняется свойство $(36)$ и $\sigma \in \mathscr{K}_{2}(T)$.

Аналогично рассматривается случай умножения на $\lambda_{2}(y)$. Теорема доказана.

Подобно тому, как с помощью теоремы 1 доказьваются теоремы 2,3 и лемма 3 для кратных рядов Уолша, с помошью теоремы 10 доказываются теоремы 11, 12 и лемма 10. Мы сформулируем все эти утверждения и дадим доказательство первой из теорем. Следующий принцип локализации для суммируемых функций дает

ТеОрема 11. Пусть $f$ и $g$ - суммируемые на $[0,2 \pi)^{2}$ функиии, рядь Фурье которых $S(f)$ и $S(g)$ принадлежсат $\mathscr{K}_{2}(T)$. Eсли $f$ и $g$ совпадают на некотором открытом множестве $G$, то $S(f)$ и $S(g)$ равносходятся на $G$.

ДоказАтЕльство. Пусть $\left(x_{0}, y_{0}\right) \in G$ и $\Pi=\left(a_{1}, b_{1}\right) \times\left(a_{2}, b_{2}\right)$ (где $a_{i}, b_{i} \in$ $[0,2 \pi))$ - такой прямоугольник, что $\left(x_{0}, y_{0}\right) \in \Pi \subset G$. Положим

$$
\lambda_{1}(x)=\left\{\begin{array}{ll}
1, & x \in\left(a_{1}+\varepsilon, b_{1}-\varepsilon\right), \\
0, & x \notin\left(a_{1}, b_{1}\right),
\end{array} \quad \lambda_{2}(y)=\left\{\begin{array}{l}
1, y \in\left(a_{2}+\varepsilon, b_{2}-\varepsilon\right) \\
0, y \notin\left(a_{2}, b_{2}\right)
\end{array}\right.\right.
$$

где $\varepsilon>0$ таково, что $\lambda_{1}\left(x_{0}\right)=1, \lambda_{2}\left(y_{0}\right)=1 ; \lambda_{1}(x), \lambda_{2}(y) \geqslant 0, \lambda_{1}(x)$ и $\lambda_{2}(y)$ имеют три непрерывные производные, так что их ряды $\Phi$ урье $\lambda_{1}(x)=\sum a_{\nu} e^{i \nu x}$, $\lambda_{2}(y)=\sum b_{\nu} e^{i \nu y}$ удовлетворяют условию $\sum\left|\nu a_{\nu}\right|<\infty, \sum\left|\nu b_{\nu}\right|<\infty$. УМножим ряд $S(f)$ формально на ряд Фурье $\lambda_{1}(x)$, а затем $\lambda_{2}(y)$. Тогда полученный ряд $S_{1}$ будет рядом Фурье функции $\lambda_{1}(x) \lambda_{2}(y) f(x, y)$ и будет равносходиться с рядом $\lambda_{1}(x) \lambda_{2}(y) S(f)$ в силу теоремы 10 . Аналогично определим ряд $S_{2}$, умно-

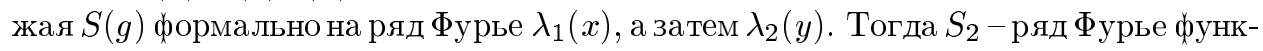
ции $\lambda_{1}(x) \lambda_{2}(y) g(x, y)$ и равносходится с $\lambda_{1}(x) \lambda_{2}(y) S(g)$. Так как $f$ и $g$ совпадают на $\Pi$, то $S_{1}=S_{2}$. Значит, ряды $\lambda_{1}(x) \lambda_{2}(y) S(f)$ и $\lambda_{1}(x) \lambda_{2}(y) S(g)$ равносходятся на $[0,2 \pi)^{2}$. В силу определения функций $\lambda_{1}$ и $\lambda_{2}$ отсюда следует, что $S(f)$ и $S(g)$ равносходятся в точке $\left(x_{0}, y_{0}\right)$, а значит, и на $G$. Теорема доказана.

Определения множеств и семейств множеств $U\left(\mathscr{K}_{2}(T)\right), M\left(\mathscr{K}_{2}(T)\right)$ даются так же, как для рядов Уолша. Аналогом теоремы Привалова для кратных тригонометрических рядов является

ТЕОРема 12. Пусть ряд $S$ класса $\mathscr{K}_{2}(T)$ сходится по прямоугольникам всюду, за исключением, быть мохсет, замкнутого $U$-множества, к конечной суммируемой на $[0,2 \pi)^{2}$ функиии $f$, причем ряд Фурве функиии $f$ тожсе класса $\mathscr{K}_{2}(T)$. Тогда $S=S(f)$.

(При доказательстве теоремы 3 мы ссылались на теорему А, а здесь доказательство опирается на аналогичную теорему Тетунашвили [5] для кратных тригонометрических рядов.) 
Лемма 10. Если $\mathscr{E}$ - ядро ряда $S$ класса $\mathscr{K}_{2}(T)$ и $\mathscr{E} \cap G \neq \varnothing$ для некоторого открытоого множества $G$, то найдется ряд класса $\mathscr{K}_{2}(T)$ с ядром $\mathscr{~} 1$, $\varnothing \neq \mathscr{E}_{1} \subset \mathscr{E} \cap G ;$ в частности, множество $\mathscr{E} \cap G$ является $M\left(\mathscr{K}_{2}(T)\right)$-множеством.

Из леммы 10 следует, что дополнительное к наследственному семейству $U\left(\mathscr{K}_{2}(T)\right)$ семейство $M\left(\mathscr{K}_{2}(T)\right)$ является семейством со свойством ядра. Отсюда и из теорем $\mathrm{F}$ и К следуют

TЕОРема 13. Пусть $E_{k}-U\left(\mathscr{K}_{2}(T)\right)$-множества, замкнутые относительно своего обвединения $E=\bigcup_{k=1}^{\infty} E_{k}$, причем $E$ имеет тип $G_{\delta}$. Тогда $E$ тоже является $U\left(\mathscr{K}_{2}(T)\right)$-множеством.

ТЕОРема 14. Пусть $E_{1}$ и $E_{2}$ являются $U$-множествами для двойных тригонометрических рядов, причем множество $[0,2 \pi)^{2} \backslash\left(E_{1} \cup E_{2}\right)$ содерэит какое-нибудь существенное множество. Тогда если

1) $E_{1}$ и $E_{2}$ - множества mипа $G_{\delta}$, а $E_{1} \cap E_{2}-$ типа $F_{\sigma}$ (в частности, nycmo)

uлu

2) $E_{1}$ - мнохсество типа $F_{\sigma}$ и $G_{\delta}$ одновременно, то $E_{1} \cup E_{2}$ является $U$-мнохеством.

Доказанные результаты допускают обобщение на $d$-кратные тригонометрические ряды $(d>2)$. При этом условия $(35)-(38)$ переходят для класса $\mathscr{K}_{d}(T)$ в $2 d$ условий, которые выполняются в $[0,2 \pi)^{d-1}$, и суммирование в которых ведется по всем, кроме одной, переменным.

\section{Список литературы}

1. Скворцов B. А. О коэффициентах сходящихся кратных рядов Хаара и Уолша // Вестн. МГУ. Сер. 1. Матем., мех. 1973. №6. С. 77-79.

2. Мовсисян Х. О. О единственности двойных рядов по системам Хаара и Уолша // Изв. АН Арм. ССР. Матем. 1974. Т. 9. №1. С. 40-61.

3. Лукомский C. Ф. О некоторых классах множеств единственности кратных рядов Уолша // Матем. сб. 1989. Т. 180. № 7. С. 937-945.

4. Ash J.M., Welland G. V. Convergence, uniqueness and summability of multiple trigonometric series // Trans. Amer. Math. Soc. 1972. V. 163. № 1. P. 401-436.

5. Тетунашвили III. Т. О некоторых функциональных рядах и решение проблемы единственности кратных тригонометрических рядов для сходимости по Прингсхейму // Матем. сб. 1991. Т. 182. № 8 . С. $1158-1176$.

6. Голубов Б.И., Ефимов А.В., Скворцов В.А. Ряды и преобразования Уолша. М.: Наука, 1987.

7. Привалов И. И. Обобщение теоремы P. DuBois-Reymond'a // Матем. сб. 1923. Т. 31. № 2. С. 229-231.

8. Скворцов B. A. Некоторые обобщения теоремы единственности для рядов по системе Уолша // Матем. заметки. 1973. Т. 13. №3. С. 367-372.

9. Холщевникова H. H. Теоремы об объединении $U$-множеств // Матем. заметки. 2000. T. 67. № 5. C. 778-787.

10. Сквориов B. А., Талалян A. А. Некоторые вопросы единственности кратных рядов по системе Хаара и тригонометрической системе // Матем. заметки. 1989. Т. 46. № 2. C. 104-113.

11. Куратовский К. Топология. Т. 1. М.: Мир, 1966. 
12. Бари Н. К. Тригонометрические ряды. М.: Физматгиз, 1961.

13. Kahane S. Antistable classes of thin sets in harmonic analysis // Illinois J. Math. 1993. V. 37. P. $186-223$.

14. Konyagin S. V. Every set of resolution is an Arbault set // C. R. Acad. Sci. Paris. Sér. I Math. 1992. V. 314. P. 101-104.

15. Холщевникова Н. Н. О теореме Кантора-Лебега для кратных тригонометрических рядов // Фундамент. физико-матем. проблемы . . . Сб. научных трудов. 2000. № 3. С. 35-40.

Московский государственный

технологический университет "Станкин"

E-mail: kholsh@stanmat.mian.su
Поступила в редакцию 31.05.2001 